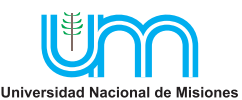

Vol. $2 \mathrm{~N}^{\circ} 2$, julio-diciembre 2020, pag. $72-80$

ISSN 2618-5520 on line

DOI - j.masingenio.2021.02.02.006

\title{
Cinética del cambio de color en snacks de mandioca fritos por inmersión
}

\author{
Hase S.L.* a , Linares A ${ }^{a}$, Kachuk L. ${ }^{a}$, Blanco M. ${ }^{a}$, Perez Navarro O ${ }^{b}$, Miño Valdés J.E. ${ }^{c}$ \\ ${ }^{a}$ Facultad de Ciencias Exactas Químicas y Naturales, Universidad Nacional de Misiones (UNaM), Posadas, Misiones. \\ ${ }^{b}$ Facultad de Química y Farmacia, Universidad Central de las Villas (UCLV), Santa Clara, Cuba \\ ${ }^{c}$ Facultad de Ingeniería, Universidad Nacional de Misiones(UNaM), Oberá, Misiones, Argentina. \\ e-mails: sandra.hase2011@gmail.com, arlinares2011@gmail.com, leonardokachuk@gmail.com, \\ martinblanco1@gmail.com,jemino53@hotmail.com
}

\section{Resumen}

El objetivo de este trabajo fue modelar la cinética del cambio de color, de snacks de mandioca, durante su fritura por inmersión a 150,170 ó $190^{\circ} \mathrm{C}$. Los snacks preparados a partir de puré de mandioca extruidos fueron fritos en aceite de girasol y su cambio de color medido con colorímetro Hunter Lab D25-9. La luminosidad (L) disminuyó exponencialmente mientras que el parámetro redness ("a") y el cambio de color total ( E) aumentaron con el incremento del tiempo de fritura; se utilizó una ecuación de primer orden para modelarlos. El parámetro yellowness ("b") después de un incremento inicial, disminuyó linealmente con el tiempo de fritura. Los datos de contenido del contenido de agua de los snacks fueron ajustados a una relación empírica. La dependencia de la constante de velocidad del cambio de color total con la temperatura fue modelada mediante la ecuación de Arrhenius y su energía de activación disminuyó con la disminución del contenido de agua en el snacks.
\end{abstract}

Palabras Clave - Cinética, Color, Fritura, Mandioca.

\begin{abstract}
The objective of this work was to model the kinetics of the color change, of cassava snacks, during its frying process by immersion at 150,170 or $190^{\circ} \mathrm{C}$. The snacks prepared from extruded cassava puree were fried in sunflower oil and their color change measured with Hunter Lab D25-9 colorimeter. The luminosity (L) decreased exponentially while the redness parameter ("a") and the total color change ( E) increased with increasing frying time. A first order equation was used for modeling. The parameter yellowness ("b") after an initial increase, decreased linearly with the frying time. The water content data of the snacks were adjusted to an empirical relationship. The dependence of the speed constant of the total color change with temperature was modeled by the Arrhenius equation and its activation energy decreased with the reduction in the water content in the snacks.
\end{abstract}

Keywords - Kinetic, Color, Frying, Cassava. Snacks

\section{Introducción}

La mandioca es un producto regional que se consume hervida, o hervida y luego frita, cuya transformación industrial en nuevos productos alimenticios es casi nula, destinándose principalmente a la producción de fécula. La industrialización de la mandioca en la provincia de Misiones se realizaba casi exclusivamente mediante la extracción de almidón nativo y su conversión a un conjunto de almidones modificados y a algunos adhesivos para diferentes tipos de aplicaciones. La producción de puré deshidratado de mandioca, es una nueva aplicación desarrollada con la colaboración del grupo de investigadores en la FCEQyN de la UNaM e industrializada en la Cooperativa Agrícola e Industrial San Alberto Ltda, (Puerto Rico, Misiones) 
La formulación de un snack a partir del puré de esta raíz es una buena alternativa para ampliar la aplicación industrial de esta materia prima debido a las ventajas de reproducibilidad, uniformidad y pérdida de defectos y al rol que cumplen estos alimentos crujientes en la dieta de los consumidores modernos [1]. El color de los productos de snack fritos es uno de los factores de calidad más significativos para su aceptación.

Los extrudidos de puré de mandioca son blancos y el color se genera durante la fritura debido a cambios físicos y químicos causados por los fenómenos de transferencia de masa y calor que tienen lugar durante la operación [2], [3]; por lo tanto, la temperatura del aceite y el tiempo de fritura afectan el color de los snacks de mandioca.

Se han realizado muchos estudios de la cinética de cambio de color para productos fritos por inmersión profunda en aceite pero escasos trabajos sobre snacks de puré de mandioca.

Se han reportado los parámetros cinéticos de cambio de color para albóndigas de carne [4], papas fritas [2], [5], tofu [3] y Gulabjamun [6]; en ellos se señala la influencia de: la temperatura y composición del aceite, el tiempo de fritura y el tamaño de la muestra.

El objetivo de este trabajo fue modelar la cinética del cambio de color en los snacks de puré de mandioca, durante la fritura por inmersión a 150,170 o $190^{\circ} \mathrm{C}$.

\section{Materiales y métodos}

Los snaks de mandioca se prepararon a partir de puré obtenido por compresión de raíces de mandioca cocidas al vapor, variedad Rocha, de la cosecha anual 2013, provistas por la Cooperativa Agrícola San Alberto Ltda. y conservadas a $-24^{\circ} \mathrm{C}$. Los extruidos se obtuvieron haciendo pasar el puré a través de orificios de $6 \mathrm{~mm}$ de diámetro y se conservaron en Ziploc herméticos a $-24^{\circ} \mathrm{C}$ hasta la realización de las experiencias. Los mismos fueron fritos por 60, 120, 180, 240, 300, 360 y 540 segundos para evaluar pérdida de agua, absorción de aceite y color.

Para la fritura por inmersión se utilizó una freidora marca Moulinex ${ }^{\circledR}$ de 1,2 $\mathrm{kg}$ de capacidad con control de temperatura. La temperatura del aceite se controló mediante una termocupla con sonda Testo de inmersión flexible estanca tipo $\mathrm{K}$ clase 1 , rango de $\left(-60\right.$ a 1.000) ${ }^{\circ} \mathrm{C}$, transductor patrón Testo un canal T/P modelo 925, con tiempo de respuesta 2 segundos. La freidora se cargó con 2,2 L de aceite de girasol marca Natura ${ }^{\circledR}$ y se mantuvo una relación snacks/aceite de 0,2 (\% peso/volumen). La temperatura de fritura se fijó en: 150, 170 ó $190{ }^{\circ} \mathrm{C}$. Los snacks fritos fueron removidos del aceite mediante una cesta extraíble provista por la freidora y colocados sobre papel adsorbente. Después de cada experiencia se controló el volumen de aceite, el que fue cambiado después de cada hora de fritado. Todas las experiencias se realizaron por triplicado y los resultados se presentan como un promedio de los valores individuales obtenidos.

El agua contenida en los snacks fritos fueron medidos por secado en estufa a $100^{\circ} \mathrm{C}$ hasta peso constante y el contenido de aceite en los snacks fritos fueron determinados mediante extracción con Twisselman. Las muestras secas fueron molidas y extraídas con éter de petróleo (p.e. $60-80{ }^{\circ} \mathrm{C}$ ) por 4 horas. El éter de petróleo fue removido por evaporación y los matraces de Twisselman, colocados a $60{ }^{\circ} \mathrm{C}$ en estufa por 24 hs. El aceite recuperado fue pesado por triplicado, los resultados son el 
promedio de los valores obtenidos. Las muestras de snacks molidas fueron utilizadas para la medición de color. Los parámetros de color $(\mathrm{L}, \mathrm{a}, \mathrm{b})$ fueron medidos con un colorímetro HunterLab D25-9 (Hunter Associates Laboratory, Reston VA). Las muestras fueron escaneadas en ocho diferentes localizaciones para determinar los valores de L, a, b como el promedio de las ocho mediciones.

\subsection{Modelos cinéticos}

El contenido de agua (Ws) y el contenido de aceite (Fs) de los snacks de mandioca se expresan en base al contenido de sólidos no grasos, a partir de los valores expresados en base seca (W y F, respectivamente), como sigue:

$$
\begin{aligned}
W_{S} & =\frac{W}{1-W-F} \\
F_{S} & =\frac{F}{1-W-F}
\end{aligned}
$$

Donde: $W$ y $F$ son el contenido de agua y el contenido de aceite de los snacks, expresados en ( $\mathrm{kg} / \mathrm{kg}$ masa de snacks en base seca), y $W_{S}$ y $F_{S}$ son el contenido de agua y el contenido de aceite de los snacks, expresados en ( $\mathrm{kg} / \mathrm{kg}$ masa de materia seca no grasa $)$.

La cantidad de agua perdida se modela mediante la ecuación [5]:

$$
W_{S}=\left(W_{S_{0}}^{(1-n)}+(1-n) q\right)^{1 /(1-n)}
$$

Donde $W_{S_{0}}$ es el contenido de agua inicial, $W_{S}$ es el contenido de agua al tiempo t, y $n$ y $q$ son parámetros de ajuste del modelo que se obtienen por regresión múltiple para cada temperatura de fritura.

Para describir los cambios de color de los parámetros L y "a" durante el proceso de fritura se eligió un modelo cinético de primer orden [2].

$$
\frac{d C}{d t}=-K_{c}\left(C_{0}-C_{e}\right)
$$

Donde $C$ es el parámetro de color (L, "a"), $C_{e}$ es el valor de equilibrio, $K_{c}$ es la constante de velocidad $\left(\mathrm{min}^{-1}\right)$ de cada parámetro de color, $C_{0}$ es el valor inicial para cada parámetro de color al tiempo cero y $t$ es el tiempo de fritura (min). Los parámetros $C_{e}$ y $K_{c}$ para los parámetros de color L y "a" fueron estimados por un método de regresión no lineal.

Para describir los cambios de color del parámetro " $b$ " durante el proceso de fritura se eligió un modelo cinético de orden cero. 


$$
\frac{d b}{d t}=-K_{b}
$$

Donde $b$ es el parámetro de color "b", $K_{b}$ es la constante de velocidad $\left(\min ^{-1}\right)$ del parámetro de color "b" y $t$ es el tiempo de fritura (min).

Los cambios de color fueron calculados por [5]:

$$
\Delta E=\left(\left(L-L_{0}\right)^{2}+\left(a-a_{0}\right)^{2}+\left(b-b_{0}\right)^{2}\right)^{1 / 2}
$$

Donde $L, a$ y $b$ son los parámetros de color medidos al tiempo $t$ y $L_{0}, a_{0}$ y $b_{0}$ son los parámetros de color al tiempo cero.

El cambio de color durante la fritura se ajustó a la siguiente relación empírica [2]:

$$
\Delta E=b_{1}+b_{2} \exp \left(-\frac{t}{b_{3}}\right)
$$

Donde $b_{1}, b_{2}$ y $b_{3}$ son parámetros del modelo, estimados por un método de regresión no lineal.

Los valores de la constante de velocidad específica del cambio de color, $k$, se determinaron a partir de [2]:

$$
k=\frac{-b_{2} \exp \left(-t / b_{3}\right)}{b_{3}\left(\Delta E_{\text {máx }}-\Delta E\right)}
$$

Donde es el máximo valor del cambio de color obtenido experimentalmente y $\Delta E$ es el cambio de color al tiempo $t$.

Los valores experimentales de los parámetros de color $\mathrm{L}$, a y b y los valores de contenido de agua se sometieron a un análisis estadístico con pruebas de ANOVA y pruebas de rango múltiple utilizando el Statgraphics Centurion ${ }^{\circledR}$ XVI versión 16.0.09.

La bondad del ajuste de los modelos a los datos experimentales, se evaluaron mediante el error cuadrático medio (RMSE):

$$
\text { RMSE }=\left(\frac{\sum_{i=1}^{n}\left(c_{c a l}-c_{\text {exp }}\right)_{n}^{2}}{n}\right)^{0,5}
$$

Donde $c_{c a l}$ es el valor estimado por el modelo, $c_{\text {exp }}$ es el valor obtenido experimentalmente y $n$ es el número de datos experimentales. 


\section{Resultados y discusión}

\subsection{Cinética de pérdida de agua}

El contenido inicial de agua en las muestras de $\mathrm{Ws}_{0}=0,593$ disminuye rápidamente con el tiempo de fritura. Los valores experimentales de Ws se ajustaron al modelo dado por la ecuación (3). Los valores de los parámetros cinéticos n y q y de la bondad del ajuste del modelo se resumen en la Tabla 1 y los valores experimentales y los predichos por el modelo se presentan en la Fig. 1.

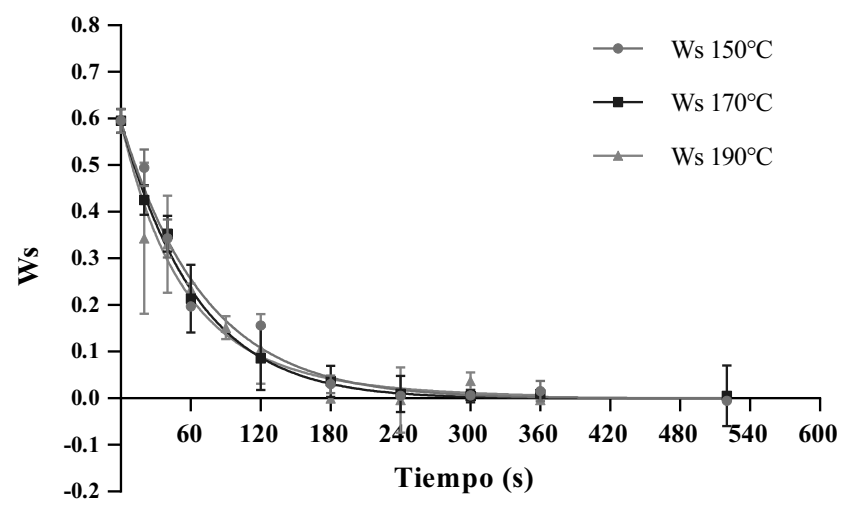

Fig. 1: Datos experimentales y modelo cinético para la pérdida de agua de snacks de mandioca por fritura a $150{ }^{\circ} \mathrm{C}, 170^{\circ} \mathrm{C}$ ó $190^{\circ} \mathrm{C}$

Tabla 1: Valores de los parámetros cinéticos n y q para la ecuación (3) y la bondad del ajuste del modelo para la pérdida de agua

\begin{tabular}{lccc}
\hline $\mathrm{T}\left({ }^{\circ} \mathrm{C}\right)$ & $\mathrm{n}$ & $\mathrm{q}\left(\mathrm{s}^{-1}\right)$ & ${ }^{*} \mathrm{R}^{2}$ \\
\hline 150 & $0,974 \pm 0,109$ & $-0,014 \pm 0,001$ & 0,980 \\
170 & $0,945 \pm 0,076$ & $-0,015 \pm 0.001$ & 0,991 \\
190 & $1,161 \pm 0,151$ & $-0,151 \pm 0,004$ & 0,956 \\
\hline
\end{tabular}

*R: coeficiente de determinación

\subsection{Luminosidad (L)}

Un análisis de ANOVA indica que tanto el tiempo como la temperatura de fritura influyen significativamente sobre el parámetro de color $\mathrm{L}(\mathrm{p}<0,05)$. Los valores experimentales de la luminosidad (L) se ajustaron al modelo de primer orden dado por la ecuación (4). Los valores de los parámetros cinéticos $\mathrm{L}_{\mathrm{e}} \mathrm{y} \mathrm{K}_{\mathrm{L}} \mathrm{y}$ de la bondad del ajuste del modelo se resumen en la Tabla 2 y los valores experimentales y los predichos por el modelo se presentan en la Fig. 2.

El valor inicial de luminosidad (L) de los snacks de mandioca fritos es de 73,01 $\pm 1,13$. Este valor disminuye con el tiempo de fritura como se muestra en la Fig. 2. El cambio es aparentemente rápido en las primeras etapas de fritura y luego se vuelve más lento hasta alcanzar los valores de equilibrio estimados, que dependen de la temperatura del aceite, según se muestra en la Tabla 2. Un comportamiento similar fue reportado en tofu [3] y en nuggets de pollo [7]. A medida que aumenta la temperatura de fritura la luminosidad disminuye para el mismo tiempo de fritura, y los valores de luminosidad de equilibrio decrecen. Esto puede atribuirse a reacciones de caramelización 
a altas temperaturas de fritura [7]. Considerando que el oscurecimiento es un efecto no deseado en un producto frito, serían aconsejables cortos tiempos de fritura a bajas temperaturas.

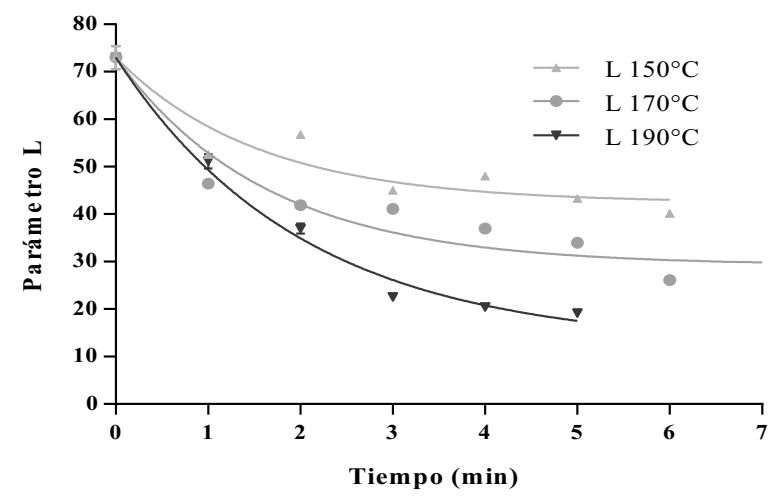

Fig. 2: Datos experimentales y modelo cinético de primer orden para la luminosidad de snacks de mandioca obtenidos por fritura a 150,170 y $190{ }^{\circ} \mathrm{C}$

Tabla 2: Valores de los parámetros cinéticos $\mathrm{L}_{0}, \mathrm{~L}_{\mathrm{e}}$ y $\mathrm{K}_{\mathrm{L}}$ para el modelo de primer orden y la bondad del ajuste del modelo para la luminosidad (L)

\begin{tabular}{lcccc}
\hline $\mathrm{T}\left({ }^{\circ} \mathrm{C}\right)$ & $\mathrm{L}_{\mathrm{e}}$ & $\mathrm{K}_{\mathrm{L}}\left(\mathrm{min}^{-1}\right)$ & $* \mathrm{R}^{2}$ & $* * \mathrm{RMSE}$ \\
\hline 150 & $42,39 \pm 0,70$ & $0,645 \pm 0,049$ & 0,814 & 3,99 \\
170 & $29,23 \pm 0,63$ & $0,614 \pm 0,031$ & 0,885 & 4,28 \\
190 & $12,43 \pm 1,02$ & $0,435 \pm 0,021$ & 0,976 & 2,81 \\
\hline \multicolumn{5}{l}{ *R: coeficiente de determinación. **Error cuadrático medio }
\end{tabular}

\subsection{Parámetro " $a$ ”}

Un análisis de ANOVA indica que tanto el tiempo como la temperatura de fritura influyen significativamente sobre el parámetro de color "a" $(p<0,05)$. Los valores experimentales del parámetro "a" se ajustaron al modelo de primer orden dado por la ecuación (4). Los valores de los parámetros cinéticos $\mathrm{a}_{0}, \mathrm{a}_{\mathrm{e}} \mathrm{y} \mathrm{K}_{\mathrm{a}} \mathrm{y}$ de la bondad del ajuste del modelo se resumen en la Tabla 3 y los valores experimentales y los predichos por el modelo se presentan en la Fig. 3.

Como se muestra en la Fig. 3 el parámetro "a" de los snacks de mandioca fritos aumentan con el tiempo de fritura a partir de un valor inicial de - 0,74 $\pm 0,05$, indicando que los productos se vuelven más rojo. A medida que aumenta la temperatura de fritura los valores de "a" de equilibrio decrecen.

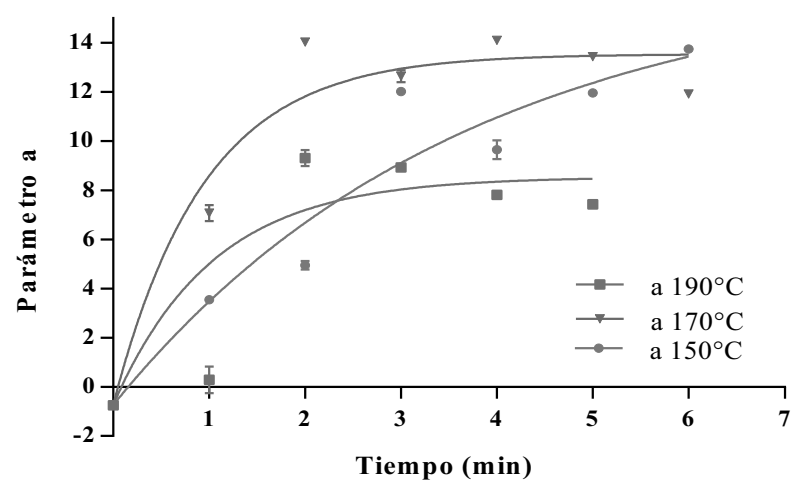

Fig. 3: Datos experimentales y modelo cinético de primer orden para el parámetro "a" de snacks de mandioca obtenidos por fritura a $150{ }^{\circ} \mathrm{C}, 170^{\circ} \mathrm{C}$ y $190^{\circ} \mathrm{C}$ 
Tabla 3: Valores de los parámetros cinéticos $\mathrm{a}_{0}, \mathrm{a}_{\mathrm{e}} \mathrm{y} \mathrm{K}_{\mathrm{a}}$ para el modelo de primer orden y la bondad del ajuste del modelo para el parámetro "a"

\begin{tabular}{ccccc}
\hline $\mathrm{T}\left({ }^{\circ} \mathrm{C}\right)$ & $\mathrm{a}_{\mathrm{e}}$ & $\mathrm{K}_{\mathrm{a}}\left(\mathrm{min}^{-1}\right)$ & $* \mathrm{R}^{2}$ & $* * \mathrm{RMSE}$ \\
\hline 150 & $16,82 \pm 0,89$ & $0,274 \pm 0,026$ & 0,900 & 1,48 \\
170 & $13,13 \pm 0,14$ & $1,139 \pm 0,063$ & 0,897 & 1,35 \\
190 & $8,54 \pm 0,97$ & $0,965 \pm 0,146$ & 0,757 & 1,78 \\
\hline \multicolumn{5}{c}{ *R: coeficiente de determinación. **Error cuadrático medio }
\end{tabular}

\subsection{Parámetro " $b$ "}

Un análisis de ANOVA indica que tanto el tiempo como la temperatura de fritura influyen significativamente sobre el parámetro de color " $b$ " $(p<0,05)$. El valor inicial del parámetro " $b$ " de los snacks de mandioca fritos fue de 8,61 $\pm 0,17$. Este valor aumentó inicialmente con el tiempo de fritura hasta el máximo color dorado de 23,90 $\pm 0,05\left(150^{\circ} \mathrm{C}, 2\right.$ minutos $), 20,43 \pm 0,10\left(170{ }^{\circ} \mathrm{C}, 1\right.$ minuto), y $16,96 \pm 0,26\left(190^{\circ} \mathrm{C}, 1\right.$ minuto $)$ y luego disminuye hasta el tiempo final de fritura, como se observa en la Fig. 4. Los valores del parámetro "b" de esta segunda etapa de descenso se ajustaron al modelo de orden cero dado por la ecuación (5). Los valores de los parámetros cinéticos $\mathrm{b}_{0}$ y $\mathrm{K}_{\mathrm{b}} \mathrm{y}$ de la bondad del ajuste del modelo se resumen en la Tabla 4 y los valores experimentales y los predichos por el modelo se presentan en la Fig. 4. Un comportamiento similar fue reportado durante la fritura de tofu [3].

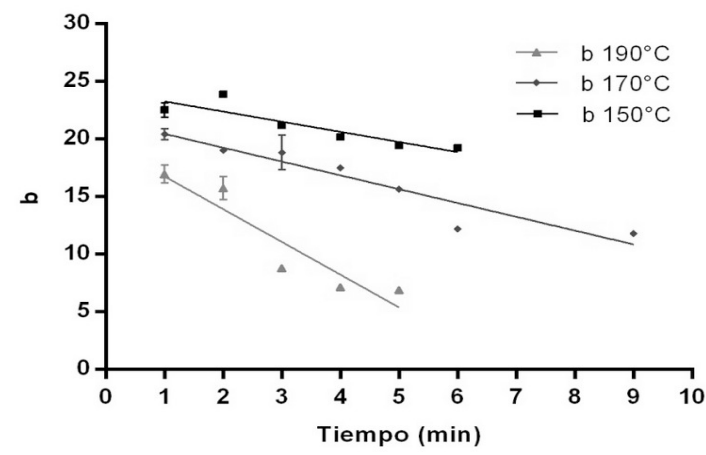

Fig. 4: Datos experimentales y modelo cinético de orden cero para el parámetro "b" de snacks de mandioca obtenidos por fritura a $150{ }^{\circ} \mathrm{C}, 170{ }^{\circ} \mathrm{C} \mathrm{y} 190^{\circ} \mathrm{C}$

Tabla 4: Valores de los parámetros cinéticos $b_{0}$ y $K_{b}$ para el modelo de orden cero y la bondad del ajuste del modelo para el parámetro "b"

\begin{tabular}{lccc}
\hline $\mathrm{T}\left({ }^{\circ} \mathrm{C}\right)$ & $\mathrm{b}_{0}$ & $\mathrm{~K}_{\mathrm{b}}\left(\mathrm{min}^{-1}\right)$ & $\mathrm{R}^{2}$ \\
\hline 150 & $24,17 \pm 0,15$ & $-0,880 \pm 0,039$ & 0,776 \\
170 & $21,67 \pm 0,18$ & $-1,200 \pm 0,037$ & 0,869 \\
170 & $19,63 \pm 0,47$ & $-2,843 \pm 0,134$ & 0,814 \\
\hline
\end{tabular}

\subsection{Cinética del cambio de color $(\Delta E)$}

Los valores experimentales del cambio de color $(\Delta E)$ calculados por la ecuación (6), se ajustaron al modelo empírico dado por la ecuación (7). Los valores de los parámetros cinéticos b1, 
b2 y b3 y de la bondad del ajuste del modelo se resumen en la Tabla 5 y los valores experimentales y los predichos por el modelo se presentan en la Fig. 5.

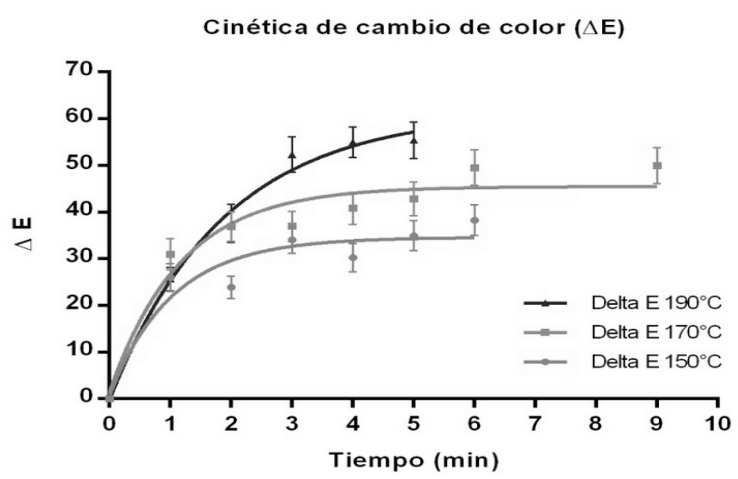

Fig. 5: Datos experimentales y modelo cinético empírico para el cambio de color $(\triangle E)$ de snacks de mandioca obtenidos por fritura a 150,170 ó $190^{\circ} \mathrm{C}$

Tabla 5: Valores de los parámetros cinéticos $b_{1}, b_{2}$ y $b_{3}$ y la bondad del ajuste del modelo para el cambio de color $(\triangle E)$

\begin{tabular}{ccccc}
\hline $\mathrm{T}\left({ }^{\circ} \mathrm{C}\right)$ & $\mathrm{b}_{1}$ & $\mathrm{~b}_{2}$ & $\mathrm{~b}_{3}$ & $* \mathrm{R}^{2}$ \\
\hline 150 & $34,56 \pm 0,56$ & $-33,72 \pm 1,01$ & $1,04 \pm 0,08$ & 0,872 \\
170 & $45,45 \pm 0,57$ & $-44,26 \pm 1,07$ & $1,17 \pm 0,07$ & 0,904 \\
190 & $61,42 \pm 1,27$ & $-61,63 \pm 1,36$ & $1,87 \pm 0,11$ & 0,967 \\
\hline \multicolumn{5}{c}{ *R coeficiente de determinación }
\end{tabular}

Para obtener los parámetros cinéticos del color se procede como sigue: para cada valor de Ws dado y para cada temperatura de fritura, se obtiene el tiempo para alcanzar dicho contenido de agua (Fig.1). Así, para un dado contenido de agua y tiempo de reacción se determina el valor de (EA) (Fig. 5) para cada temperatura. A partir de estos valores se determina k (constante de velocidad específica del cambio de color) con la ecuación (8), haciendo posible obtener un gráfico de Arrhenius para determinar la energía de activación $\left(\mathrm{E}_{\mathrm{a}}\right)$ para valores de contenido de agua seleccionados. En la Tabla 6 se dan valores de energía de activación para el cambio de color como función del contenido de agua

Tabla 6: Energía de activación para el cambio de color $(\Delta E)$ a contenidos de agua seleccionados

\begin{tabular}{cc}
\hline $\mathrm{Ws} \%$ & $\mathrm{E}_{\mathrm{a}}(\mathrm{kJ} / \mathrm{mol})$ \\
\hline 59,26 & 16,05 \\
50 & 15,66 \\
40 & 13,97 \\
30 & 9,80 \\
20 & 0,35 \\
\hline
\end{tabular}


La energía de activación $\mathrm{E}_{\mathrm{a}}(\mathrm{kJ} / \mathrm{mol})$ disminuyó de $(16,05$ a 0,35) a medida que disminuyó el contenido de humedad Ws \% de (59,26 a 20$)$.

\section{Conclusiones}

El cambio de color fue modelado usando ecuaciones cinéticas de 1er orden para la luminosidad (L) y el parámetro de color redness "a"; mientras que el parámetro de color yellowness "b" fué ajustado con una ecuación de orden cero.

El valor de "a" aumentó con el Tiempo de fritado volviéndose mas rojizo y a mayor Tiempo de fritado los valores de "a"

El Tiempo y la Temperatura de fritado influyeron significativamente, a un nivel de confianza del 95\%, sobre los parámetros de color redness y yellowness.

La cinética modelada se puede aplicar para predecir cambios de color en función del contenido de agua, cuando los snacks de mandioca son fritados en aceite de girasol a 150,170 ó $190^{\circ} \mathrm{C}$.

\section{Referencias}

[1] J. M. Barata de Carvalho, "Textura de snacks crujientes culinarios", tesis doctoral, Universidad de Extremadura, Cáceres, España, 2012.

[2] M. K. Krokida, V. Oreopoulou, Z. B. Maroulis, D. Marinos-Kouris, "Colour changes during deep fat frying", Journal of Food Engineering, vol. 48, pp. 219-225, 2001.

[3] O. D. Baik y G. S. Mittal, "Kinetics of tofu color changes during deep-fat frying”, Lebebsm.-Wiss. U.Technol., vol. 36, pp. 43-48, 2003.

[4] P. Ateba y G. S. Mittal, "Dynamics of crust formation and kinetics of quality changes during frying of meatballs", Journal of Food Science, vol. 59, pp. 1275-1278, 1994.

[5] P. C. Moyano, V. K. Ríoseco, P. A. González, "Kinetics of crust color changes during deep-fat frying of impregnated french fries", Journal of Food Engineering, vol. 54, pp. 249-255, 2002.

[6] A. Jayendra Kumar, R. R. B. Singh, A. A. Patel, G. R. Patil, "Kinetics of color and texture changes in Gulabjamun balls during deep-fat frying”, LWT, vol. 39, pp. 827-833, 2006.

[7] L. Yunsheng, "Quality changes in chicken nuggets fried in oils with different degrees of hydrogenation", Thesis for the degree of Master of Science, McGill University, Montreal, Quebec, Canadá, 2005. 